\title{
Qualidade de vida de graduandos de enfermagem
}

\author{
Quality of life of undergraduate nursing students
}

Calidad de vida de estudiantes del grado de enfermería

\section{Beatriz Marques de Oliveira', Vivian Aline Mininel', Vanda Elisa Andres Felli' \\ 'Universidade de São Paulo. Escola de Enfermagem de Ribeirão Preto. Ribeirão Preto, SP}

Submissão: 1 1/09/2009

Aprovação: 14/08/2010

\section{RESUMO}

O presente estudo objetivou descrever o perfil sociodemográfico dos estudantes de enfermagem e compreender o significado atribuído para sua atual Qualidade de vida. Foram entrevistados 65 acadêmicos do $8^{\circ}$ semestre do ano de 2007, por meio da aplicação de Questionário autoreferido, com informações de dados sociodemográficos e Questões abertas sobre Qualidade de vida. A população de estudo foi predominantemente do sexo feminino, solteira, com idade entre 22 a 24 anos e com diferentes percepções sobre sua Qualidade de vida, apreendidas por meio da vivência de fatores favoráveis e desfavoráveis no decorrer dos anos de aprendizado. O estudo destacou a percepção da Qualidade de vida sob a ótica do estudante de enfermagem.

Descritores: Qualidade de vida; Estudantes de enfermagem; Enfermagem.

\section{ABSTRACT}

The present study aimed to describe socio-demographic profile of nursing students and to comprehend the meaning of Quality of life under theirs perspective. Sixty-five students of eighth semester of 2007 were interviewed, by application of self reported Questionnaire, with Questions about socio demographic data and open Question about Quality of life. The population was mainly female, single, with age among 22 to 24 years old and with different perceptions about own Quality of life, apprehended by experience of favorable and destructive factors during the learning years. The study highlighted the Quality of life perception of nursing student's perspective.

Key words: Quality of life; Students, nursing; Nursing.

\section{RESUMEN}

El objetivo del presente estudio fue describir el perfil sociodemográfico de los estudiantes de enfermería y comprender sus percepciones sobre propia calidad de vida. Sesenta y cinco estudiantes del octavo semestre de 2007 fueran entrevistados por el uso del cuestionario, con informaciones sobre datos sociodemográficos y preguntas abiertas sobre calidad de vida. La populación era principalmente femenina, solas, con edad entre 22 y 24 años y con diversas opiniones sobre propia calidad de vida, prendida por la experiencia factores favorables e destructibles durante los años de la aprendizaje. El estudio destacó la percepción de la calidad de vida de los estudiantes de la enfermería. Descriptores: Calidad de vida;, Estudiantes de enfermería; Enfermería. 


\section{INTRODUÇÃO}

A Qualidade de vida $(\mathrm{QV})$ é um termo associado aos vários aspectos relacionados ao ser humano, em proporções subjetivas atribuídas por cada indivíduo, de acordo com sua perspectiva de análise. Por este motivo, até o momento, tal expressão não possui uma definição consensual Que consiga representá-la em sua totalidade ${ }^{(\mathrm{l})}$. Este termo carrega uma noção eminentemente humana, Que tem sido aproximada ao grau de satisfação encontrado na vida familiar, amorosa, social, ambiental e à própria estética existencial ${ }^{(2)}$.

Por estar intrinsecamente conectada aos aspectos de saúde, por diversas vezes, a QV é utilizada como sinônimo de saúde. Apesar da visível relação entre as duas condições, afirmar Que Qualidade de vida é ter saúde é reduzi-la a um único fragmento de bem-estar da integralidade humana.

Nesta perspectiva, uma crescente importância tem sido atribuída à Questão da Qualidade de vida inerente ao conceito de saúde, não mais restrita à ausência de doença, porém composta por, pelo menos, seis domínios: o físico, o psicológico, o nível de independência, as relações sociais, o meio ambiente e a espiritualidade ${ }^{(3)}$. De acordo com tais premissas, a Qualidade de vida pode ser definida como "a percepção do indivíduo de sua posição na vida no contexto da cultura e sistema de valores nos Quais ele vive e em relação aos seus objetivos, expectativas, padrões e preocupações"(4).

Dois aspectos constantes na definição acima serão explorados neste estudo: a percepção individual sobre Qualidade de vida e o trabalho como sendo parte indissociável da vida humana e Que contempla todos os aspectos supramencionados. A QV tem sido referida tanto no momento da vida dos indivíduos em sociedade, como no momento de trabalho, entendendo Que estes se constroem mutuamente. Esta afirmação está pautada no entendimento de Que não há como dissociar a vida e o trabalho, bem como de não reconhecer a interface do trabalho na $\mathrm{QV}^{(5)}$.

Apesar da percepção individual da QV resultar da evolução histórica e social de cada ser humano, as considerações realizadas acerca dos aspectos relacionados ao trabalho pactuam do mesmo ambiente físico e organizacional, o Que possibilita a construção de um perfil de Qualidade de vida para o grupo. Assim, mesmo sendo percebida em nível individual, a QV decorre de uma estrutura articulada no macro contexto social, sendo Que as ações individuais só alcançam representatividade Quando projetadas para o nível coletivo ${ }^{(6)}$.

Sob os pressupostos da Epidemiologia Social, apreende-se a Qualidade de vida como sendo gerada na reprodução social, Que conforma processos favoráveis e desgastantes no momento de vida social e de trabalho, Que ora potencializam a saúde e a vida e ora a doença e a morte. É o perfil epidemiológico dos grupos sociais que expressa a Qualidade de vida desse grupo ${ }^{(7)}$.

Assim, a construção do conceito de QV, gerada na Reprodução Social, deve basear-se na contextualização dos aspectos positivos e negativos presentes na vida e no trabalho do ser humano. Sabidamente, a avaliação da Qualidade de vida é uma ferramenta importante para compreender como o estilo de vida atual pode exercer influência sobre a saúde e, conseQuentemente, repercutir na Qualidade de vida dos indivíduos ${ }^{(8)}$.

Todavia, estudos sobre a Qualidade de vida dos estudantes universitários e, mais especificamente, estudantes de enfermagem tem sido escassos na literatura nacional e internacional. Em contrapartida, as pesQuisas com trabalhadores de enfermagem e riscos ocupacionais envolvidos têm obtido crescente destaque no meio científico. Esta realidade demonstra a preocupação com a Qualidade de vida no trabalho dos profissionais de enfermagem, fato Que não acontece nos anos precursores à vida profissional ativa.

Um estudo realizado com estudantes universitários definiu a Qualidade de vida como a percepção de satisfação e felicidade, por parte do estudante, em relação aos múltiplos domínios de vida à luz de fatores psicossociais e contextuais relevantes e estruturas de significados pessoais. Estes domínios foram apontados como: social, individual, acadêmico, gênero, finanças, moradia, serviços da universidade, administração universitária, e outros (entre eles, saúde). A relevância da Qualidade de vida durante os anos de graduação justifica sua inclusão como uma importante variável nos esforços de compreensão das experiências e resultados atribuídos pelos estudantes no processo vivido em sua formação( ${ }^{(9)}$.

Outro relevante estudo sobre esta temática pautou-se no modelo ecológico para analisar acadêmicos de enfermagem. Este aponta a universidade como espaço Que proporciona vivências promotoras e não promotoras da Qualidade de vida dos alunos ${ }^{(10)}$.

Entende-se por acadêmico ou estudante de enfermagem o ser humano Que faz uma opção por cuidar e ajudar outros seres humanos a nascer e viver de forma saudável; a superar agravos à sua saúde; a conviver com limitações e encontrar um significado nessa experiência e; a morrerem com dignidade. No processo de preparar-se para realizar as várias ações Que integram esse trabalho, com competência técnica, dialógica e política, enfrenta situações de sofrimento Que podem contribuir tanto para processo de humanização deste trabalho, Quanto para sua banalização(II).

Outros estudos têm mostrado Que a exposição dos estudantes de enfermagem às cargas de trabalho se assemelham a exposição dos profissionais já inseridos no mercado de trabalho ${ }^{(12-13)}$. Esta semelhança sinaliza para um futuro profissional com os mesmos comprometimentos Que hoje acometem os trabalhadores de saúde, caso uma intervenção não seja aplicada ainda na vida acadêmica desta categoria profissional.

Neste contexto, este estudo propõe compreender a Qualidade de vida sob a perspectiva dos acadêmicos de enfermagem, por meio da exploração dos processos favoráveis e desgastantes presentes na formação profissional.

\section{MÉTODOS}

Tratou-se de um estudo descritivo-exploratório de recorte Qualitativo, apoiado no referencial teórico da epidemiologia social. O cenário de pesquisa foi uma escola pública e universitária de enfermagem, da cidade de São Paulo. A amostra foi composta por 65 acadêmicos de enfermagem regularmente matriculados no $8^{\circ}$ semestre do ano de 2007. A amostra foi intencional, uma vez que o estudo buscou apreender os dados da vivência cumulativa destes alunos durante os Quatro anos de formação, acreditando-se Que possuíam melhores oportunidades de avaliação da Qualidade de vida durante toda a formação.

Previamente à fase de coleta de dados, o projeto foi encaminhado ao Comitê de Ética e Pesquisa da Universidade Que aprovou a realização do estudo, bem como a coleta de dados (Processo 50/ 2007). A participação dos estudantes ocorreu de forma voluntária, 
formalizada por meio da assinatura individual do Termo de Consentimento Livre e Esclarecido ${ }^{(14)}$.

A coleta de dados foi realizada por meio da aplicação de um Questionário auto-referido, composto por informações sociodemográficas e Questões sobre a Qualidade de vida dos estudantes de enfermagem. As duas dimensões objetivaram, respectivamente, a caracterização da população e a compreensão dos significados e percepções sobre a Qualidade de vida atual desta categoria. Também foi utilizada uma escala de 0 a 10 , no Qual o 0 significava a pior Qualidade de vida e o 10 a melhor Qualidade de vida.

Todos os estudantes Que participaram da coleta de dados receberam os esclarecimentos necessários acerca do objetivo da pesquisa, da garantia de sigilo e anonimato, do caráter voluntário da participação e da possibilidade de desistência, sem incidência de prejuízos. Após tal ciência, os participantes assinaram o Termo de Consentimento Livre e Esclarecido, confirmando sua participação voluntária na pesquisa. Os alunos foram convidados a preencher o Questionário Quando estavam todos reunidos em sala de aula, no início do segundo semestre de 2007. Os Questionários preenchidos foram devolvidos pessoalmente para a pesquisadora.

Os dados sociodemográficos foram introduzidos em planilhas Excel e foram analisados segundo frequências absolutas e relativas. Os dados Qualitativos foram submetidos à análise temática, segundo temas estabelecidos no decorrer da apreciação ${ }^{(15)}$.

No procedimento de análise temática dos dados obtidos, foi realizada a leitura flutuante do conteúdo, Que consiste na realização de uma leitura extensiva do material até a impregnação do mesmo. Em seguida, a análise temática desenvolveu-se na tentativa de captar os "núcleos dos sentidos" contidos nas falas, cuja presença ou frequência tem algum significado importante para os sujeitos Que vivenciam determinadas circunstâncias presentes no dia a dia ${ }^{(15)}$.

Após a análise temática, os temas Que emergiram das falas foram agrupados nas seguintes categorias: significado da Qualidade de vida; fatores que favorecem a Qualidade de vida e; fatores que comprometem a Qualidade de vida. A avaliação da Qualidade de vida atual foi realizada por meio da interpretação dos resultados contidos na escala gradual de Qualidade de vida. Na apresentação dos discursos, os acadêmicos foram identificados por números cardinais, observando-se o princípio ético do anonimato.

\section{RESULTADOS}

\section{Caracterização sociodemográfica}

Dos 78 acadêmicos regularmente matriculados no $8^{\circ}$ semestre do curso de enfermagem no ano de 2007, 65 dispuseram-se a participar do estudo (83\%). As informações sociodemográficas obtidas por meio da aplicação do Questionário autoreferido são descritas na Tabela I.

Serão apresentadas, a seguir, as principais categorias Que emergiram da análise temática, elaboradas a partir dos temas recorrentes nas respostas dos Questionários, remetendo-se às citações textuais dos estudantes para cada categoria proposta.

\section{Significado da Qualidade de vida}

As falas abaixo demonstram a associação entre a Qualidade de vida e a satisfação das necessidades humanas básicas:

“(...) ter saúde, moradia, família, acesso a meios de transporte de
Qualidade, acesso à educação, ter emprego, acesso à atividade de lazer e cultura, alimentar-se com Qualidade, dormir em tempo necessário para um descanso adequado". (Estudante I)

“(...) organizar o dia, separando um tempo adequado para a família, estudo, trabalho, alimentação, atividades físicas e relacionamentos sociais (...)" (Estudante 2)

A satisfação das expectativas individuais, bem como a somatória e fatores favoráveis e desfavoráveis no processo de viver, também foram referidas como Qualidade de vida:

“(..) conseguir trabalhar suas perspectivas de vida de modo a sentir-se satisfeito e atingir suas expectativas e as Que não forem atingidas serem trabalhadas internamente afim de não produzirem frustrações e desmotivação". (Estudante 3)

“(..) perpassa por determinantes Que podem ser de fortalecimento e de desgaste e que fluem no modo de viver, a Qualidade pode ser a média na relação entre estes potenciais de fortalecimento $e$ desgaste". (Estudante 4)

Tabela 1. Características sociodemográficas dos estudantes $(\mathrm{N}=65)$, São Paulo, SP, 2007.

\begin{tabular}{|c|c|c|}
\hline Características & $\mathrm{n}$ & $\%$ \\
\hline \multicolumn{3}{|l|}{ Sexo } \\
\hline Feminino & 60 & 92,31 \\
\hline Masculino & 5 & 7,69 \\
\hline \multicolumn{3}{|l|}{ Faixas etárias } \\
\hline 21 a 22 anos & 26 & 40,00 \\
\hline 23 a 24 anos & 26 & 40,00 \\
\hline 25 a 26 anos & 9 & 13,85 \\
\hline 27 a 28 anos & 3 & 4,62 \\
\hline 29 anos ou mais & 1 & 1,54 \\
\hline \multicolumn{3}{|l|}{ Estad o civil } \\
\hline Solteiro & 59 & 90,77 \\
\hline Casado & 5 & 7,69 \\
\hline Amasiado & 1 & 1,54 \\
\hline \multicolumn{3}{|l|}{ Flhos } \\
\hline Não & 64 & 98,46 \\
\hline Sim & 1 & 1,54 \\
\hline \multicolumn{3}{|l|}{ Co- residên daa } \\
\hline Com a família & 57 & 87,69 \\
\hline Com amigo(s) & 6 & 9,23 \\
\hline Sozinho & 2 & 3,08 \\
\hline \multicolumn{3}{|c|}{ Meio de transporte até à Universidade } \\
\hline Trem / metrô & 20 & 30,77 \\
\hline Ônibus & 17 & 26,15 \\
\hline Ônibus + metrô/trem/a pé & 10 & 26,15 \\
\hline Carro próprio & 10 & 15,38 \\
\hline Moto & 1 & 1,54 \\
\hline \multicolumn{3}{|c|}{ Tempo de percurso até à Universidade } \\
\hline Até 30 minutos & 9 & 13,85 \\
\hline 31 a 45 minutos & 10 & 15,38 \\
\hline 46 a 60 minutos & 12 & 18,46 \\
\hline Acima de 60 minutos & 34 & 52,31 \\
\hline \multicolumn{3}{|l|}{ Atividade remunerada } \\
\hline Sim & 27 & 41,54 \\
\hline Não & 38 & 58,46 \\
\hline
\end{tabular}


Alguns acadêmicos definiram Qualidade de vida relacionando-a a fatores Que geram prazer e bem estar:

"Exercer tarefas que dão prazer, satisfação e vontade de realizálas, sem estresse..." (Estudante 5)

\section{Fatores Que favorecem a Qualidade de vida}

Os estudantes apontaram diversos fatores Que favorecem a Qualidade de vida dentro da Universidade. As falas abaixo destacam alguns fatores Que favorecem a QV e o impacto destes na saúde:

"A interação com as pessoas e as amizades conquistadas, a construção de conhecimento e a ampliação da visão de mundo, os benefícios de ser aluno desta universidade e estudante (bandejão, bilhete único, acesso à biblioteca com retirada de livros)". (Estudante 6)

“(...) melhoram a minha auto-estima, a minha alimentação, alterando a minha disposição de uma forma positiva, o Que aumenta a minha resistência contra doenças oportunistas..." (Estudante 7)

\section{Fatores Que comprometem a Qualidade de vida}

As falas abaixo apontaram alguns fatores Que comprometem a Qualidade de vida, bem como a influência de tais fatores na saúde dos estudantes:

"Aulas pouco didáticas, estágios exigentes, alta cobrança por parte dos professores, falta de ética com os alunos, professores inflexíveis, prazos curtos de entrega de trabalhos, sobrecarga em geral". (Estudante 8)

“(...) me provocam estresse, irritação e desconsolo, desencadeando problemas como enxaqueca, gastrites e doenças de pele". (Estudante 9)

\section{Avaliação da Qualidade de vida atual}

A avaliação da Qualidade da vida atual foi realizada por meio da tabela gradual de 0 a 10 , onde 0 significava a pior Qualidade de vida e 10 a melhor Qualidade de vida. Os gradientes variaram de 2 a 9 , conforme distribuição na Tabela 2.

Tabela 2. Classificação da eualidade de vida atual, referida pelos estudantes. São Paulo, SP, 2007:

\begin{tabular}{lcc}
\hline Classificação da QV & $\mathbf{n}$ & $\%$ \\
\hline 0 & - & 0,00 \\
1 & - & 0,00 \\
2 & 1 & 1,54 \\
3 & 2 & 3,08 \\
4 & 5 & 7,69 \\
5 & 4 & 6,15 \\
6 & 15 & 23,08 \\
7 & 19 & 29,23 \\
8 & 15 & 23,08 \\
9 & 4 & 6,15 \\
10 & - & 0,00 \\
\hline Total & 65 & 100,00 \\
\hline
\end{tabular}

\section{DISCUSSÃO}

A caracterização sociodemográfica demonstrou Que $92,31 \%$ dos participantes do estudo são do sexo feminino, reproduzindo a força de trabalho em Enfermagem enquanto profissão, Que ainda é predominantemente feminina ${ }^{(16)}$. Estudos relacionados ao perfil sociodemográfico dos estudantes de enfermagem encontraram percentuais semelhantes, o Que corrobora com o resultado acima mencionado ${ }^{(17-21)}$. Os dados encontrados neste e em outros estudos reforçam duas tendências já constatadas pela literatura; uma delas, Quanto à relação historicamente construída entre a mulher e o cuidado e a outra, relacionada à relação_socialmente construída entre a mulher e a opção pelos cursos de enfermagem ${ }^{(22)}$.

Em relação à idade, houve o predomínio das faixas etárias dentre 21 a 24 anos, com 52 estudantes (80,00\%). Somente um aluno classificou-se na faixa etária superior a 29 anos; este estudante tinha 40 anos de idade. Esse dado revela um perfil bastante jovem dos estudantes, peculiar nas universidades públicas do País. Isto também mostra o Quão precocemente estes profissionais poderão se inserir no mercado de trabalho, uma vez que o presente estudo avalia egressos de enfermagem. Estes percentuais convergem com os resultados de um estudo realizado com ingressos do curso de enfermagem em duas instituições de ensino no município do Rio de laneiro, Que evidenciou 48\% da população nas faixas etárias entre 17 e 19 anos de idade ${ }^{(23)}$.

Os dados evidenciam que a maioria dos estudantes é solteira e não possui filhos, perfil bastante coerente com a média de idade dos estudantes. Ambos percentuais são semelhantes a um estudo realizado no interior do Estado de São Paulo, no Qual aproximadamente $90 \%$ dos estudantes de enfermagem eram solteiros e não possuíam filhos $^{(24)}$.

Grande parte dos peseuisados mora com a família $(87,69 \%)$ e utilizam transportes coletivos (83\%) para chegar à Universidade e aos locais de estágio, sendo Que mais da metade deles demoram mais de uma hora para percorrer tais percursos. Estas informações evidenciam situações desgastantes Que a maioria dos estudantes enfrenta para se locomover até o local onde estuda, fato Que impacta diretamente no declínio da eualidade de vida.

Quanto aos dados referentes à atividade remunerada, 27 estudantes referiram possuir alguma atividade remunerada, como bolsa de iniciação cientifica, bolsa trabalho, estágio remunerado, monitoria, trabalho formal ou trabalho autônomo. Um outro estudo com população semelhante revelou um dado bastante interessante: $40 \%$ dos estudantes de enfermagem recebiam mesada da família, o Que salientou a importância do suporte familiar para o desenvolvimento dos estudos ${ }^{(19)}$. Este item não foi referido pelos participantes da presente pesquisa, possivelmente, por não se enQuadrar como atividade remunerada e, sim, como uma outra forma de subsídio financeiro.

Os graduandos atribuíram diversos significados para a Qualidade de vida, de acordo com as perspectivas e vivências individuais, apreendidas durante toda a vida. Muitas falas relacionam a Qualidade de vida à satisfação das necessidades humanas básicas, indispensáveis para uma vida digna. É inegável Que, para se ter Qualidade de vida, primeiramente necessita-se das condições mínimas de desenvolvimento enQuanto ser humano; satisfeitas as necessidades primárias, surge a percepção de Qualidade de vida como sendo "fazer o que se 
gosta”, independente de QualQuer outra razão(6).

Assim, outras falas representaram a percepção de Qualidade de vida como "poder fazer o que gostam" e "terem tempo para o trabalho e para a vida pessoal (família, lazer e o cuidado de si)". A Qualidade de vida pode ser definida como a percepção do indivíduo de sua posição na vida, no contexto da cultura e sistema de valores nos Quais vive e em relação aos seus objetivos, expectativas, padrões e preocupações ${ }^{(25)}$.

A percepção dos estudantes também foi ao encontro da abordagem da Qualidade de vida gerada na reprodução social, Que conforma processos favoráveis e desgastes, no momento de vida social e de trabalho, Que ora potencializam a saúde e a vida e ora a doença e a morte, resultando em padrões de saúde-doença $\mathrm{a}^{(26)}$.

Dentre os fatores Que favorecem a Qualidade de vida dentro da universidade, os estudantes destacaram os laços de amizade estabelecidos com os colegas, a alimentação oferecida por preço acessível, o grande acervo da biblioteca, o conhecimento técnico adQuirido, a boa convivência com funcionários e professores e o fato da universidade ser pública. Além disso, os participantes também destacaram fatores não relacionados diretamente ao ensino, como participação em atividades do centro acadêmico e centro de práticas esportivas; possibilidade de obtenção de bolsas de iniciação científica, bolsa-trabalho e outros auxílios; a facilidade de acesso à universidade e a infraestrutura oferecida.

A promoção de relaxamento, o alívio da tensão, o aumento da autoconfiança, da autoestima e do bem-estar, a melhora do padrão de sono e o aumento da resistência às doenças oportunistas foram aspectos relacionados à prevalência dos fatores favoráveis na vida universitária.

Quanto aos fatores Que comprometem a Qualidade de vida, os mais relatados foram sobrecarga de atividades, distância diária percorrida entre a residência e a universidade, período integral do curso, falta de tempo para atividades extracurriculares, desorganização das disciplinas, aulas pouco didáticas, relacionamento conflituoso com os docentes, alto grau de exigência durante o período dos estágios curriculares, curtos prazos para entrega de trabalhos, falta de ética profissional, competitividade entre os alunos, gastos financeiros e falta de espaço e tempo para o lazer.

Os graduandos mencionaram Que tais fatores provocam estresse, desgaste físico e mental, dores no corpo, desânimo, ansiedade, cefaléia e enxaQueca, Queda da resistência imunológica, gripes e resfriados, irritação, desinteresse, gastrite, entre outros, sendo o estresse mais referido pelos estudantes. Um estudo Que relaciona o estresse e a enfermagem demonstrou Que os estudantes apresentam sintomas Que caracterizam o estresse negativo, resultante da falha dos mecanismos de adaptação a situações estressantes, o Que resulta em perda da Qualidade de vida, alteração nos padrões de sono, aparecimento de dores e irritabilidade ${ }^{(27)}$.

Quando Questionados, os estudantes relataram Que tanto os fatores favoráveis Quanto os comprometedores da Qualidade de vida impactam diretamente em sua saúde, sendo os aspectos negativos aqueles Que mais prevalecem no cotidiano e, assim, adQuirem maiores proporções na vida e saúde.

Para avaliação da Qualidade de vida atual, foi solicitado aos estudantes Que circulassem os gradientes de uma escala de 0 a 10 , considerando 0 a pior Qualidade de vida e 10 a melhor Qualidade de vida. A atribuição dos valores para a Qualidade de vida pode ter sido influenciada pela ansiedade gerada pelo término do curso ou pelo desenvolvimento de estratégias redutoras de estresse e promotoras de prazer, desenvolvidas ao longo do curso.

Do total de estudantes avaliados, 12,3\% atribuíram gradientes mais baixos para sua Qualidade de vida (nota 5 ou menos), relacionandoos ao cansaço, a realização da monografia, atividade remunerada concomitante com a faculdade, sobrecarga de atividades e estresse. Dentre aqueles que atribuíram nota 6 e entre 6 e 7 (24,6\% da amostra) alegaram, além das causas supramencionadas, a ansiedade pela conclusão do curso, prazos curtos para cumprimento das atividades, falta de tempo para o lazer, problemas familiares, e pressão sofrida pelo início da carreira profissional.

A maior parte dos acadêmicos $(50,7 \%)$ atribuiu notas entre 7 e 8 para sua Qualidade de vida atual. Alguns consideram essas notas altas como resultado da sensação de bem-estar, estratégias individuais de relaxamento, enfrentamento do estresse, término da graduação, atividades extracurso com familiares, maior flexibilidade na grade horária. Nove foi à nota mais elevada atribuída por alguns estudantes $(6,2 \%)$, justificando-a pela flexibilidade de tempo para realizar outras atividades, por atuar na área Que gosta e por participar de algum tipo de religião. Um estudo sobre a Qualidade de vida de estudantes de enfermagem conforme escala de Flanagan também evidenciou Que a maioria dos estudantes está satisfeita com sua Qualidade de vida e com o curso, mas também possibilitou detectar a presença de sofrimento, ansiedade, necessidade de atenção e cuidado ${ }^{(28)}$.

Sob esta visão, dentre os estudantes Que consideraram Que a nota poderia ser melhor do Que a atribuída, mas não 10 , relacionaram-na a desmotivação, ansiedade, cansaço, tempo elevado no percurso entre residência e universidade, preocupações com o término da graduação e sobrecarga de atividades.

A transição entre a vida de estudante e profissional assume formas e problemas específicos em cada contexto, consoante às exigências colocadas pelas características de desenvolvimento dos próprios estudantes e dos cursos $^{(10)}$. Esta transição implica em uma necessidade de ajuste social e emocional ou a aQuisição das estratégias de coping, necessárias para fazer face aos novos papéis, entre outras exigências ${ }^{(29)}$. As estratégias de coping são esforços para administrar ou alterar um problema, ou melhorar o relacionamento entre a pessoa e seu meio. São estratégias adaptativas, Que podem estar dirigidas ao ambiente Quando relacionadas às fontes externas de estresse, ou dirigidas ao próprio indivíduo - busca de suportes sociais e materiais ${ }^{(30)}$.

Estas estratégias de enfrentamento possibilitam aos estudantes e também profissionais de enfermagem a gestão dos fatores Que comprometem a $\mathrm{QV}$, para Que representem menor impacto nos aspectos da vida laboral e social.

\section{CONCLUSÕES}

Este estudo demonstrou Que a maioria dos acadêmicos de enfermagem, na universidade estudada, é do sexo feminino, solteira, tem idade entre 22 a 24 anos, não possui filhos, mora com os pais, utiliza-se de transporte urbano para chegar à universidade, não exerce atividade remunerada e demora mais de 60 minutos no percurso entre sua casa e a universidade.

Os resultados evidenciaram Que os acadêmicos têm diferentes percepções sobre Qualidade de vida e Que existem fatores Que favorecem e comprometem Qualidade de vida durante os anos de formação. Estes fatores configuram o perfil de saúde e doença desta 
população, Que é determinado ora pela prevalência dos fatores positivos, Que promovem a Qualidade de vida e saúde, ora por fatores negativos, Que a degradam. Os fatores comprometedores da Qualidade de vida foram destacados nas falas dos estudantes, Que referiram especialmente o estresse como conseQuência desta exposição.

De uma forma geral, a maioria dos entrevistados diz-se satisfeita com sua Qualidade de vida atual, apesar do misto de sentimentos desencadeados pelo término da graduação, Que são geradores de ansiedade e prazer.

\section{REFERÊNCIAS}

I. Maciel ES. Qualidade de vida: análise da influência do consumo de alimentos e estilo de vida [dissertação]. Piracicaba: Escola Superior de Agricultura Luiz de Queiroz, Universidade de São Paulo; 2006

2. Minayo MCS, Hartz ZMA, Buss PM. Qualidade de vida e saúde: um debate necessário. Cien Saude Coletiva 2000; 5(1)

3. WHOQOL Group. The World Health Organization Quality of Life Assessment: position paper from the World Health Organization, 1995. Soc Sci Med 1995; 10: 1403-9.

4. WHOQOL Group. The development of the World Health Organization Quality of life assessment instrument (WHOQOL). In: Orley J, Kuyken W editors. Quality of life assessment: internationa perspectives. Heidelberg: Springer Verlag; 1994. p. 4 I-60.

5. Felli VEA, Tronchin DMR. A qualidade de vida no trabalho e a saúde do trabalhador de enfermagem. In: Kurcgant P, coordenadora. Gerenciamento em Enfermagem. Rio de Janeiro: Guanabara-Koogan; 2005. p. 89-107.

6. Mininel VA. Promoção da Qualidade de vida dos trabalhadores de enfermagem: responsabilidade gerencial do enfermeiro [dissertação]. São Paulo: Escola de Enfermagem, Universidade de São Paulo; 2006.

7. Breilh I, Granda E. Investigação da saúde na sociedade: guia pedagógico sobre um novo enfoque do método epidemiológico. São Paulo: ABRASCO; 1986.

8. Oliveira JAC. Qualidade de vida e desempenho acadêmico de graduandos [tese]. Campinas: Faculdade de Educação, Universidade Estadual de Campinas; 2006.

9. Benjamin M. The Quality of student life: toward a coherent conceptualization. Soc Indic Res 1994; 31 : 205-64.

10. Oliveira RA. A universidade como espaço promotor de Qualidade de vida: vivências e expressões dos alunos de enfermagem [tese]. São Paulo: Escola de Enfermagem, Universidade de São Paulo; 2005.

11. Saupe R, Nietche EA, Cestari ME, Giorgi MDM, Krahl M. Qualidade de vida dos acadêmicos de enfermagem. Rev Latinoam Enfermagem 2004; 12(4).

12. Barachati VS. A exposição dos graduandos de enfermagem aos fluídos corpóreos durante a realização dos estágios [monografia]. São Paulo: Escola de Enfermagem, Universidade de São Paulo; 2005.

13. Scudeler LA. Exposição dos Graduandos de Enfermagens às Cargas Químicas [monografia]. São Paulo: Escola de Enfermagem, Universidade de São Paulo; 2006.

14. Ministério da Saúde (BR). Conselho Nacional de Saúde. Resolução N. 196/96 sobre pesquisas envolvendo seres humanos. Bioética 1996; 4(2): 15-25

15. Minayo CSM. O desafio do conhecimento. $9^{\mathrm{a}}$ ed. São Paulo: Hucitec; 2006
Assim, é importante que a universidade esteja consciente de seu papel na formação não somente técnica, mas também sociocultural do enfermeiro e de como isso irá refletir no início da carreira profissional destes alunos. Estratégias promotoras da Qualidade de vida, por meio do desenvolvimento de fatores Que a favorecem, devem ser encorajadas entre docentes, alunos e demais trabalhadores da instituição, com o intuito de proporcionar melhores condições de enfrentamento das incertezas futuras Que serão vivenciadas Quando do egresso da universidade.

16. Almeida MCP, Rocha SMM, organizadoras. O trabalho de enfermagem. São Paulo: Cortez; 1997.

17. Brito AMR, Brito MIM, Silva PAB. Perfil sociodemográfico de discentes de enfermagem de instituições de ensino superior de Belo Horizonte. Esc Anna Nery Rev Enferm 2009; I3(2): 328-33.

18. Hermida PMV. Representação social dos discentes de enfermagem sobre a profissão e profissional enfermeiro. Rev Educ 2008: II(12): 137-55.

19. Oguisso T, Lira PS, Vieira APM, Pereira KCM, Mesquita MMC Silva PJP. Perfil do estudante ingressante no curso de graduação da Escola de Enfermagem da Universidade de São Paulo. Rev Paul Enferm 2006; 25(2): 109-16.

20. Santos CE, Leite MMJ. O perfil do aluno ingressante em uma universidade particular da cidade de São Paulo. Rev Bras Enferm 2006; 59(2)

21. Wetterich NC, Melo MRAC. Perfil sociodemográfico do aluno do curso de graduação em enfermagem. Rev Lat-am Enfermagem 2007; 15(3)

22. Teixeira E, Vale EG, Fernandes ID, Sordi MRL. Trajetória e tendências dos cursos de enfermagem no Brasil. Rev Bras Enferm 2006; 59(4)

23. Spíndola T, Martins ERC, Francisco MTR. Enfermagem como opção: perfil de graduandos de duas instituições de ensino. Rev Bras Enferm 2008; 6 I (2).

24. Kawakame PMG, Miyadahira AMK. Qualidade de vida de estudantes de graduação em Enfermagem. Rev Esc Enferm USP 2005; 39(2)

25. WHOQOL GROUP. The World Health Organization Quality of Life. Assessment: development and general psychometric properties. Soc Sci Med 1998; 46(I 2): 1569-85.

26. Breilh J. La salud enfermedad como hecho social: un nuevo enfoeue. In: Breilh J. Deterioro de la vida: un instrumento para análisis de prioridades regionales in lo social y la salud. Quito: CEAS/Corporación/Editora Nacional; 1990.

27. Mauro MYC, Santos CC, Oliveira MM, Lima PT. O estresse e a prática de enfermagem: Quando parar e refletir? Uma experiência com estudantes de enfermagem. Acta Paul Enf 2000; I 3(número especial, parte II):44-48.

28. Saupe R. A qualidade de vida de estudantes de enfermagem conforme escala de Flanagan. Cienc Saude Cuidado 2002; I (2): 287-9l.

29. Santos SM. As responsabilidades da Universidade no acesso ao Ensino Superior. In: Soares AP, editor. Transição para o ensino superior. Braga: Universidade do Minho; 2001.

30. Chaves EC, Cadê NV, Mantovani MF, Leite RCBO, Spire WC Coping: significados, interferência no processo saúde-doença e relevância para a enfermagem. Rev Esc Enferm USP 2000; 34(4). 